\title{
A study of efficacy of oral probiotics in management of cases with symptomatic white discharge per vagina in a tertiary care hospital
}

\author{
Pillai L ${ }^{1}$, Sadhya ${ }^{2}$, Narmadha N.S ${ }^{3}$, Reddy A.S. ${ }^{4}$ \\ ${ }^{1}$ Dr Lekshmi Pillai, Junior Resident, ${ }^{2}$ Dr Sadhya, Junior Resident, ${ }^{3}$ Dr Narmadha N.S, Senior Resident, ${ }^{4}$ Dr Alla \\ Satyanarayana Reddy, Professor and HOD; all authors are affiliated with Department of Obstetrics and Gynaecology, \\ Vinayaka Mission's Medical College \& Hospital, Karaikal, Puducherry.
}

Address for Correspondence: Dr Alla Satyanarayana Reddy, Professor and HOD, Department of Obstetrics and Gynaecology, Vinayaka Mission's Medical College, Karaikal, Puducherry

\begin{abstract}
Background: Urogenital infections are the most common gynecological condition constituting about $25 \%$ of outpatients. Because of higher recurrence rates and resistance to standard antimicrobials now a days the use of probiotics in augmenting normal bacterial populations is gradually achieving scientific acceptance. Objective: Determine the efficacy of probiotics in treating women with symptomatic white discharge per vagina (WDPV), role of oral probiotics in restoring the vaginal flora. Methods: 70 women aged between 18 to 45 yrs (mean age 35yrs) with symptomatic WDPV who are attending gynecology outpatient procedures, these patients underwent Grams stain, received antibiotics along with probiotics (L. rhamnosus GR-1 and L. fermentum RC-14) for a period of 1 week, again reviewed with repeat Grams stain. Results: Among post-treatment group $46 \%$ of patients showed $>30$ organisms/100× objective. Fifty percent of patients showed counts between 5 and 30 among post-treatment, but the response in terms of symptomatic relief was about $74 \%$. The improvement in the lactobacilli count was interpreted using Nugents scoring. Conclusion: The combination of probiotic (L. rhamnosus GR-1 and L. fermentum RC-14) is not only safe for daily use in healthy women, but it can reduce colonization of the vagina by potential pathogenic bacteria and yeast.
\end{abstract}

Keywords: Probiotics, Lactobacilli, Urogenital infections

\section{Introduction}

Urogenital infections afflict an estimated few billion women in a year, the size of this problem and the increased prevalence of multidrug resistant pathogens make it imperative for alternate treatment. The microorganisms that colonize the vagina play a major role in maintenance of resistance against infestations from pathogenic organisms.

When this flora is dominated by lactobacilli or a commensal flora, the person is regarded as being healthy in terms of the urogenital tract, unless other specific disease traits are evident.

When the vault is colonized primarily or solely by pathogenic bacteria, such as Escherichia coli or Gardnerella vaginalis [1-3] the patient is generally regarded as having an abnormal flora. Antimicrobial

Manuscript received: $06^{\text {th }}$ September 2016

Reviewed: $15^{\text {th }}$ September 2016

Author Corrected: $20^{\text {th }}$ September 2016

Accepted for Publication: $30^{\text {th }}$ September 2016

Obsgyne Review: Journal of Obstetrics and Gynecology therapy has been reasonably effective at curing bacterial infections of the bladder and vagina, but mounting drug resistance and failure of antibiotics to change host receptivity to pathogen recurrences, plus a negative impact on patient quality of life make it imperative that alternative therapeutics be found [4-7].

Probiotics are regarded as 'Live microorganisms which when administered in adequate amounts confer a health benefit on the host'[8]. A recent Food and Agriculture Organization of the United Nations and the World Health Organization Working Group has developed guidelines for what constitutes a true 'probiotic', and very few so called health products currently meet these criteria because they have no published clinical studies showing a benefit of their strains on the host [9]. Probiotic Lactobacillus rhamnosus GR-1 and Lactobacillus fermentum RC-14 have been shown in open studies to colonize the vagina following oral intake $[10,11]$.

Bacterial vaginosis (BV) is the most common cause of abnormal vaginal discharge in women of childbearing Available online at: www.medresearch.in 39 | P a g e 


\section{Original Research Article}

age. The causative organisms for this condition are Gardnerella vaginalis, Mycoplasma hominis and anaerobic bacteria. It is thought that a shift to a symptomatic BV state may simply be due to a decline in the levels of 'beneficial' lactic acid and hydrogen peroxide-producing lactobacilli and/or an increase in the levels of Gram-negative anaerobes. A variety of events can contribute to the development of $\mathrm{BV}$ in which a mixture of the organisms listed above is usually present in concentrations 100 to 1,000 times greater than in the healthy vagina.

The standard scoring system termed the 'Nugent score' is an accepted technique using microscopic examination of a Gram-stained smear of vaginal discharge for determining BV. Due to the wide variety of Gram smear results from vaginal samples that can be considered normal, specific bacterial types need not be reported, but may be listed as 'organisms resembling normal urogenital flora', Yeast should always be reported with an added comment, such as 'Candida species are normal flora in the genital area of 30 to $40 \%$ of women. The presence of yeast must be correlated with the clinical picture'. Smear results that score $>7$ in the Nugent scoring system should be reported as 'consistent with bacterial vaginosis'. It is acceptable to not report cells, or bacteria, and only report presence or absence of yeast, and whether smear results are consistent with BV or not [12].

Available evidence now indicates that certain strains of lactobacilli when administered to patients can colonize the vagina and reduce the risk of BV. Studies have been carried out to assess the efficacy of single strain or combination of probiotics administered orally or Intravaginally in the treatment of BV. In addition, the effect of probiotics in conjunction with antimicrobial regimen has to be evaluated. So this study is aimed to determine the efficacy of probiotics in treating women with symptomatic WDPV.

\section{Materials and Methods}

Design of study: This is a prospective randomized clinical study done on women with symptomatic white discharge per vagina (WDPV).

Settings: Symptomatic white discharge per vagina (WDPV) patients attending gynecology outpatient of Vinayaka Mission's Medical College, Karaikal, Puducherry for a period of 2 years (2014-2016).

Study population: In the present study, 70 women with history of WDPV received probiotics along with antibiotics were selected.

\section{Inclusion criteria}

- Females patients aged between 18 to 45 yrs (mean age $35 \mathrm{yrs}$ ) will be included in the study.

- Patients willing to give written informed consent.

- Clinical diagnosis was confirmed by Nugents score.

- Patients who have been newly diagnosed and/or recurrent vulvovaginitis not treated in the previous one month.

\section{Exclusion criteria}

- Pregnant or nursing women

- Patient Menstruating at the time of diagnosis

- Usage of antibacterial drugs either systemically or intravaginally within last two weeks

- Patients already on medications for vulvovaginitis.

- Patients taking immunosuppressive, or immunostimulating medications, systemic corticosteroids within 3 months prior to study.

- Patients involved in any other study in previous one month.

- Patients with comorbid conditions like diabetis mellitus and Sexually transmitted diseases (STDs) like Gonorrhea, Syphilis, Chlamydia, AIDS.

- Patients with history of allergy to metronidazole, clotrimazole, clindamycin,

Method of study: Women were examined at their first visit-after detailed history and clinical examination, including local examination to see the amount, character of discharge and for presence of cervical movement tenderness. Routine investigations to rule out presence of anemia, diabetes and urinary infection are done along with Gram stain of the vaginal discharge to study the type of organism and the load of lactobacilli, which is defined using Nugent scoring (table 1 and 2). These patients receive a combination of antibiotics (Ofloxacin + Ornidazole + fluconazole) along with probiotics containing 2,500 million spores of Lactobacilli rhamnosus GR-1 and L. fermentum RC-14, for a period of 1 week. 
Original Research Article

Table-1: Laboratory examination of vaginal smears and the determination of the Nugent score

$\mathrm{N}$ score-The sum of the scores for each bacterial morphotype listed below

\begin{tabular}{|c|c|c|c|c|c|c|}
\hline Lactobacilli & Score & $\begin{array}{c}\text { Gardnerella, } \\
\text { Bacteroides }\end{array}$ & Score & $\begin{array}{c}\text { Curved Gram-negative } \\
\text { bacilli }\end{array}$ & Score & Sum=*N score \\
\hline 30 or $>$ & 0 & 0 & 0 & 0 & 0 & 1 \\
$5-30$ & 1 & $<1$ & 1 & $<1$ & 1 & 3 \\
$1-4$ & 2 & $1-4$ & 2 & $1-4$ & 2 & 5 \\
$<1$ & 3 & $5-30$ & 3 & $5-30$ & 2 & 8 \\
0 & 4 & 30 or $>$ & 4 & 30 or $>$ & \\
\hline
\end{tabular}

Note: Number of organisms seen/100× objective

Table-2: Showing the Interpretation of Nugent score

\begin{tabular}{|c|c|c|}
\hline N-score & & Reports \\
\hline $0-3$ & Clue cells not present & Smear not consistent with BV \\
$4-6$ & Clue cells are present & Smear consistent with BV \\
$4-6$ & & \\
$>7$ & & \\
\hline
\end{tabular}

These patients are called back after 1 week and examined by the same gynecologist, asked regarding symptom relief, examination findings are compared and Grams stain is repeated to look for treatment response and also for the restoration of vaginal flora.

Statistical Analysis: Data was collected and tabulated as shown in results. Statistical analysis was done using Microsoft Excel. Frequency and percentage of each parameter was calculated and analyzed

\section{Results}

In the present study, 70 women with history of WDPV received probiotics along with antibiotics. Fifty two percent of patients were aged between 21 and 35 years indicating the increased incidence of RTI in reproductive age group. Forty-six percent had history of pain abdomen along with WDPV and the duration was between 15 days and 6 months in $74 \%$ of them.

Among them $48 \%$ had received treatment before coming here and were not relieved of the symptoms. On examination, there was copious discharge in $66,58 \%$ had cervical and vaginal congestion, $48 \%$ had erosion on the cervix.

Forty eight percent had hemoglobin levels between 8 and $10 \mathrm{gm} \%$ (mean \pm SD: $10.73 \pm 1.21$ ), this shows the correlation between anemia and vaginal infections. Four percent had random blood sugars $>160 \mathrm{mg} / \mathrm{dl}(\mathrm{mean} \pm \mathrm{SD}: 87.19 \pm 13.48), 15 \%$ of them had associated urinary tract infection.

Pretreatment Gram's stain is interpreted with respect to the lactobacilli score (Table 3) using one of the criteria's in Nugent's score.

Among patients in pretreatment group, $66 \%$ of them had $<1$ organism/100 $\times$ objective, this showed the shift in vaginal flora, only $2 \%$ of them showed count between 5 and 30 . Forty-two percent of patients showed $>30$ organisms $/ 100 \times$ in the posttreatment Grams stain. Fifty percent of patients showed counts between 5 and 30 among post-treatment. Pap smear is done for all the patients, $66 \%$ of them showed inflammatory. Seventy four (74\%) are relieved of symptoms, there was response even in terms of improved vaginal flora (Table 4).

Nine patients (18\%) were not relieved of symptoms, even though there was improvement in vaginal flora in seven of these nonresponders, they were not relieved symptomatically. The cause among these patients were non infective, i.e, chronic cervicitis in three patients, diabetes mellitus in two patients, $\mathrm{CIN}-2$ and three in two patients, one patient had $\mathrm{Cu}-\mathrm{T}$ in situ which had caused recurrent PID, one patient had history of immunocompromised status which caused recurrent pelvic inflammatory disease. 
Original Research Article

Table-3: Lactobacilli score -pre- and post-treatment

\begin{tabular}{|c|c|c|c|}
\hline & $\begin{array}{l}\text { Lactobacilli score } \\
\text { (number of organisms } \\
\text { seen } / 100 \times \text { objective) }\end{array}$ & $\begin{array}{l}\text { Pretreatment } \\
(\mathrm{n}=70)\end{array}$ & $\begin{array}{c}\text { Post-treatment } \\
(\mathrm{n}=\mathbf{7 0})\end{array}$ \\
\hline 0 & $(30$ or $>)$ & Nil & $46 \%$ \\
\hline 1 & $(5-30)$ & $2 \%$ & $50 \%$ \\
\hline 2 & $(1-4)$ & $12 \%$ & $3 \%$ \\
\hline 3 & $(<1)$ & $66 \%$ & $2 \%$ \\
\hline 4 & $(0)$ & $20 \%$ & Nil \\
\hline
\end{tabular}

Table-4: Response of treatment

\begin{tabular}{|c|c|c|}
\hline Response & Number of patients $(\mathbf{n}=\mathbf{7 0})$ & \% \\
\hline Yes & 52 & 74.0 \\
\hline
\end{tabular}

Only $32 \%$ patients had side effects like nausea, vomiting in $26 \%$, giddiness in $8 \%$ and pain abdomen in $2 \%$ of patients. But these side effects cannot be completely attributed to use of probiotics only, as in the study they receive additional antibiotics also.

\section{Discussion}

The therapy resulted in a significant improvement in the vaginal flora in terms of increased lactobacilli presence. The outcome was not designed to be mechanism-based, but the results indicate that intestinal passage of these probiotic strains led to a beneficial impact on the vaginal microflora.

This may have occurred due to the strains themselves ascending to the vagina from the rectal area. It is feasible that the therapy caused an alteration in the mucosal immunity of the host (via the gut and/ or vagina) and that this played a part in reducing pathogen counts. This study was concurrent with the previous studies. Bohbot and Cardot (2012) found that positive impact of the oral administration of LCR35 on vaginal microbiota and also decrease in the Nugent score in healthy women and therefore the maintenance of the quality of their vaginal microbiota [13].

Wagner and Johnson (2012) showed that Lactobacillus rhomnosus GR-1 and Lactobacillus reuteri RC-14 strains used in commercial products inhibit infectivity of Candida albicans the major cause of VVC [14]. Larsson et. al showed that aggressive treatment of patients with BV with antibiotics combined with lactobacillus administration can provide a long lasting cure[15].

The loss of vaginal lactobacilli appears to be the major factor in the cascade of changes leading to bacterial vaginosis and relapses are associated with failure to establish a healthy lactobacilli dominated vaginal flora.

The mode of action has not been elucidated but might comprise:

Obsgyne Review: Journal of Obstetrics and Gynecology
- Increased ascension of probiotic and/or indigenous

- lactobacilli from the rectal skin to the vagina.

- Reduced ascension of pathogens from the rectal skin to the vagina.

- Enhancement of the intestinal mucosal immunity which affects vaginal immunity rendering the environment less receptive to bacterial vaginosis organisms.

In our present study, the lactobacilli score was improved with oral probiotics. Among patients in pretreatment group, $66 \%$ of them had $<1$ organism $/ 100 \times$ objective, this showed the shift in vaginal flora, only $2 \%$ of them showed count between 5 and 30 .

Among post-treatment group $46 \%$ of patients showed $>30$ organisms $/ 100 \times$. Fifty percent of patients showed counts between 5 and 30 among post-treatment. This shows the improved vaginal flora following treatment with probiotics.

Nine patients (18\%) were not relieved of symptoms, even though there was improvement in vaginal flora in 7 of these nonresponders, they were not relieved symptomatically. The cause among these patients were non infective, i.e. chronic cervicitis in 3 patients, diabetes mellitus in 2 patients, $\mathrm{CIN}-2$ and 3 in 2 patients, one patient had $\mathrm{Cu}-\mathrm{T}$ in situ which had caused recurrent PID, one patient had history of immuno-compromised status which caused recurrent pelvic inflammatory disease.

Therefore only the altered vaginal flora leading to bacterial vaginosis should not be blamed on, as other predisposing factors has to be treated simultaneously. 
Original Research Article

Although antimicrobial therapy is generally effective in eradicating urogenital infections, there is still a high incidence of recurrence. There is good clinical evidence to show that the intestinal and urogenital microbial flora have a central role in maintaining both the health and well - being of humans.

\section{Conclusion}

This study clearly showed that combination of probiotic (L. rhamnosus GR-1 and L. fermentum RC-14) is not only secure for daily use in healthy women, but it could decrease colonization of the vagina by potential pathogenic bacteria and yeast.

Funding: Nil, Conflict of interest: Nil Permission from IRB: Yes

\section{References}

1. Wasiela M, Hanke W, Kalinka J. Association between abnormal microbiological flora of the lower genital tract in early pregnancy and socioeconomic, demographic and environmental risk factors Med Sci Monit 2001;7: $1250-55$.

2. Schwebke JR, Richey CM, Weiss2 HL. Correlation of behaviors with microbiological changes in vaginal flora. $\mathrm{J}$ Infect Dis. 1999 Nov;180(5):1632-6.

3.Bruce AW, Chadwick P, Seddon JM, Vancott GF. The significance of perineal pathogens in women. J Urol. 1974 Dec;112(6):808-10.

4. Sewankambo N, Gray RH, Wawer MJ, Paxton L, McNaim D, Wabwire-Mangen F, Serwadda D, Li C, Kiwanuka N, Hillier SL, Rabe L, Gaydos CA, Quinn TC, Konde-Lule J. HIV-1 infection associated with abnormal vaginal flora morphology and bacterial vaginosis. Lancet. 1997 Aug 23;350(9077):546-50.

5. Reid G, Bruce AW, Beheshti M. Effect of antibiotic treatment on receptivity of uroepithelial cells to uropathogens. Can J Microbiol. 1988 Mar;34(3):327-31.

6. Petrof EO, Schwartz DN, Quinn JP. Urinary tract infections and a multidrug-resistant Escherichia coli clonal group. N Eng1 J Med. 2002 Feb 14;346(7):535-6.
7. Ellis AK, Verma S. Quality of life in women with urinary tract infections: is benign disease a misnomer? J Am Board Fam Pract. 2000 Nov-Dec;13(6):392-7.

8. FAO/WHO. Evaluation of health and nutritional properties of powder milk and live lactic acid bacteria. Food and Agriculture Organization of the United Nations and World Health Organization Report 2001.

9. $\mathrm{FAO} / \mathrm{WHO}$. Guidelines for the evaluation of probiotics in food. Report of a joint FAO/WHO Working Group 2002 .

10. Reid G, Bruce AW, Fraser N, Heinemann C, Owen J, Henning B. Oral probiotics can resolve urogenital infections. FEMS Immunol Med Microbiol. 2001 Feb;30(1):49-52.

11. Reid G, Beuerman D, Heinemann C, Bruce AW. Probiotic Lactobacillus dose required to restore and maintain a normal vaginal flora. FEMS Immunol Med Microbiol. 2001 Dec;32(1):37-41.

12. Bijeta, Shreshtha Singh. Incidence of bacterial vaginosis in patients with idiopathic preterm labour. Int $\mathrm{J}$ Reprod Contracept Obstet Gynecol. 2015; 4(3): 641-646.

13. Champagne CP, Ross RP, Saarela M, Hansen KF, Charalampopoulos D. Recommendations for the viability assessment of probiotics as concentrated cultures and in food matrices. Int J Food Microbiol. 2011 Oct 3;149 (3):185-93. doi: 10.1016/j.ijfoodmicro.2011.07.005. Epub $2011 \mathrm{Jul} 14$.

14. Wagner RD, Johnson SJ. Probiotic lactobacillus and estrogen effects on vaginal epithelial gene expression responses to Candida albicans. J Biomed Sci. 2012 Jun 20;19:58. doi: 10.1186/1423-0127-19-58.

15. Larsson et al. Extended antimicrobial treatment of bacterial vaginosis combined with human lactobacilli to find the best treatment and minimize the risk of relapse.BMJ Infectious Diseases.2011;11:223.

\section{How to cite this article?}

Pillai L, Sadhya, Narmadha N.S, Reddy A.S. A study of efficacy of oral probiotics in management of cases with symptomatic white discharge per vagina in a tertiary care hospital. Obs Rev:J obstet Gynecol 2016;2(3):39-43.doi: 10.17511/joog.2016.i03.04. 